\title{
Alopecia Secondary to Hyaluronic Acid Embolization: Trichoscopic Findings
}

\author{
Daniel Asz-Sigalla Karla Iñigo-Gomez ${ }^{b} \quad$ María Fernanda Ortega-Springall ${ }^{b}$ \\ María Elisa Vega-Memije ${ }^{c}$ Roberto Arenas ${ }^{d}$ Antonella Tosti ${ }^{\mathrm{e}}$ \\ ${ }^{a}$ Oncodermatology and Trichology Clinic, National University of Mexico, Mexico City, Mexico; ${ }^{\text {b Dermatology }}$ \\ Department, "Dr. Manuel Gea Gonzalez" General Hospital, Mexico City, Mexico; 'Dermatopathology Department \\ "Dr. Manuel Gea Gonzalez" General Hospital, Mexico City, Mexico; d Micology Section, "Dr. Manuel Gea Gonzalez"

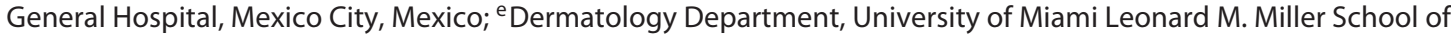 \\ Medicine, Miami, FL, USA
}

\section{Established Facts}

- Vascular occlusion leading to tissue necrosis is a rare but severe complication associated with hyaluronic acid injection.

- Alopecia secondary to hyaluronic acid injection is an underreported complication with only a couple of cases reported in the literature.

\section{Novel Insights}

- This is the first publication that describes the trichoscopic findings in hyaluronic acid induced alopecia.

- Prompt treatment of this type of alopecia leads to rapid hair regrowth, preventing the development of scarring alopecia.

\section{Keywords}

Alopecia · Hyaluronic acid · Trichoscopy

\section{Abstract}

Fillers are frequently used in aesthetic medicine and, although usually safe, complications can occur. Vascular occlusion leading to tissue necrosis is a rare but severe complication. Alopecia after hyaluronic acid injection has been recently reported, being a vascular compromise the most probable physiopathological mechanism. The trichoscopic findings in this entity have not been described yet. A case report of a 30-year-old female who developed this complication following a hyaluronic acid injection as well as the description of the trichoscopic findings are presented in this report.

(c) 2019 S. Karger AG, Basel

\section{Introduction}

Fillers are frequently used in aesthetic medicine for facial volume augmentation, correction of wrinkles, skinfolds, and scars. Although usually safe, complications can

\section{KARGER}

(c) 2019 S. Karger AG, Basel

E-Mail karger@karger.com

www.karger.com/sad
Daniel Asz-Sigall

Oncodermatology and Trichology Clinic

Universidad Nacional Autónoma de México

Emerson 132-4, Colonia Polanco, Mexico City 11560 (Mexico)

E-Mail dasck@hotmail.com 
Fig. 1. a Swelling and patchy erythema of the right forehead $48 \mathrm{~h}$ after HA injection. b Irregular alopecic patch with prominent erythema 7 days later and an erythematous linear tract along the forehead.
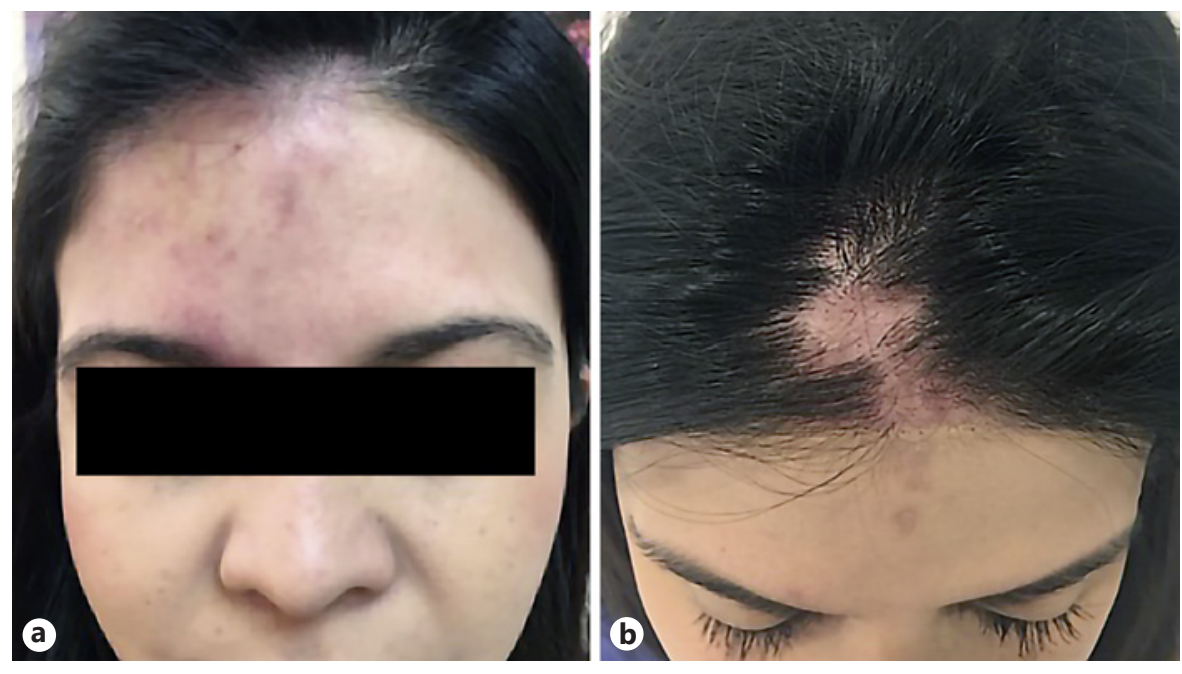

occur that include transient edema and erythema, allergic and hypersensitivity reactions, infections, granuloma formation, filler migration, and even visual impairment and blindness. Vascular occlusion leading to tissue necrosis is a rare and serious complication $[1,2]$. Alopecia after hyaluronic acid (HA) injection has been recently reported, being a vascular compromise the most probable physiopathological mechanism $[3,4]$. Trichoscopic findings of this phenomenon have not been reported.

\section{Case Presentation}

We present the case of a previously healthy 30 -year-old female who underwent botulinum toxin A injection for the treatment of frown lines, horizontal forehead lines, and crow's feet. A week after the procedure, she developed ptosis of the right eyelid. To correct this, HA was injected along the superciliary arch without any apparent complications.

Forty-eight hours later, she developed swelling of the forehead, patchy erythema, and severe pain (Fig. 1a). A soft tissue infection was suspected; thus, amoxicillin and clavulanic acid PO b.i.d. was prescribed.

A partial clinical response was observed; however, 1 week later, the patient developed a rapidly expanding patch of alopecia on the right frontoparietal region of the scalp. Hair pull test was positive at the periphery of the patch, and the skin was frankly erythematous (Fig. 1b). Intralesional triamcinolone $10 \mathrm{mg} / \mathrm{mL}$ was administered on two occasions, but the patch of alopecia continued to expand.

Trichoscopic findings showed a patch of noncicatricial alopecia with multiple yellow dots, black dots, broken hairs (Fig. 2a), and an irregular vascular proliferation composed of ectatic vessels, background erythema, and pigment deposits (Fig. 2b).

Based on the clinical history, physical examination revealing an erythematous linear tract along the forehead and the trichoscopic
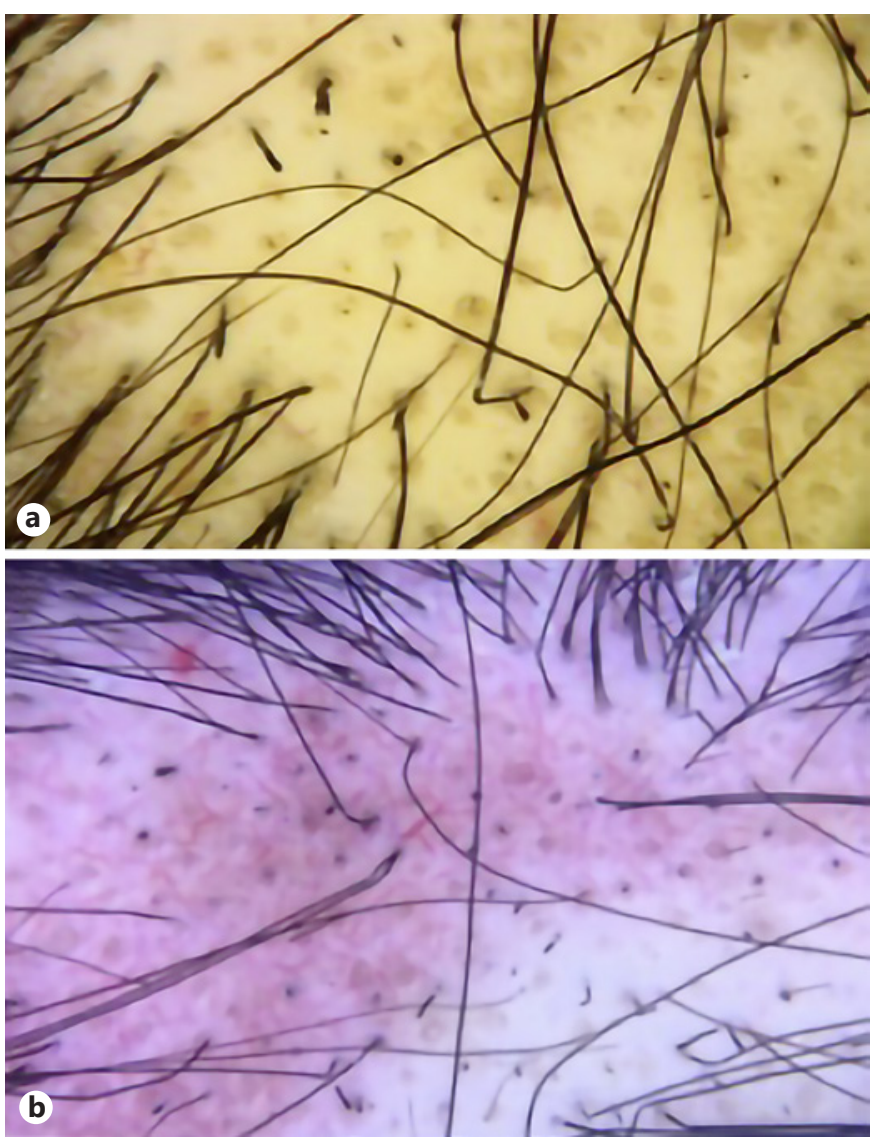

Fig. 2. a Trichoscopic images showing multiple yellow dots, black dots, and broken hairs. b Vascular proliferation composed of ectatic vessels, background erythema, and pigment deposits. 

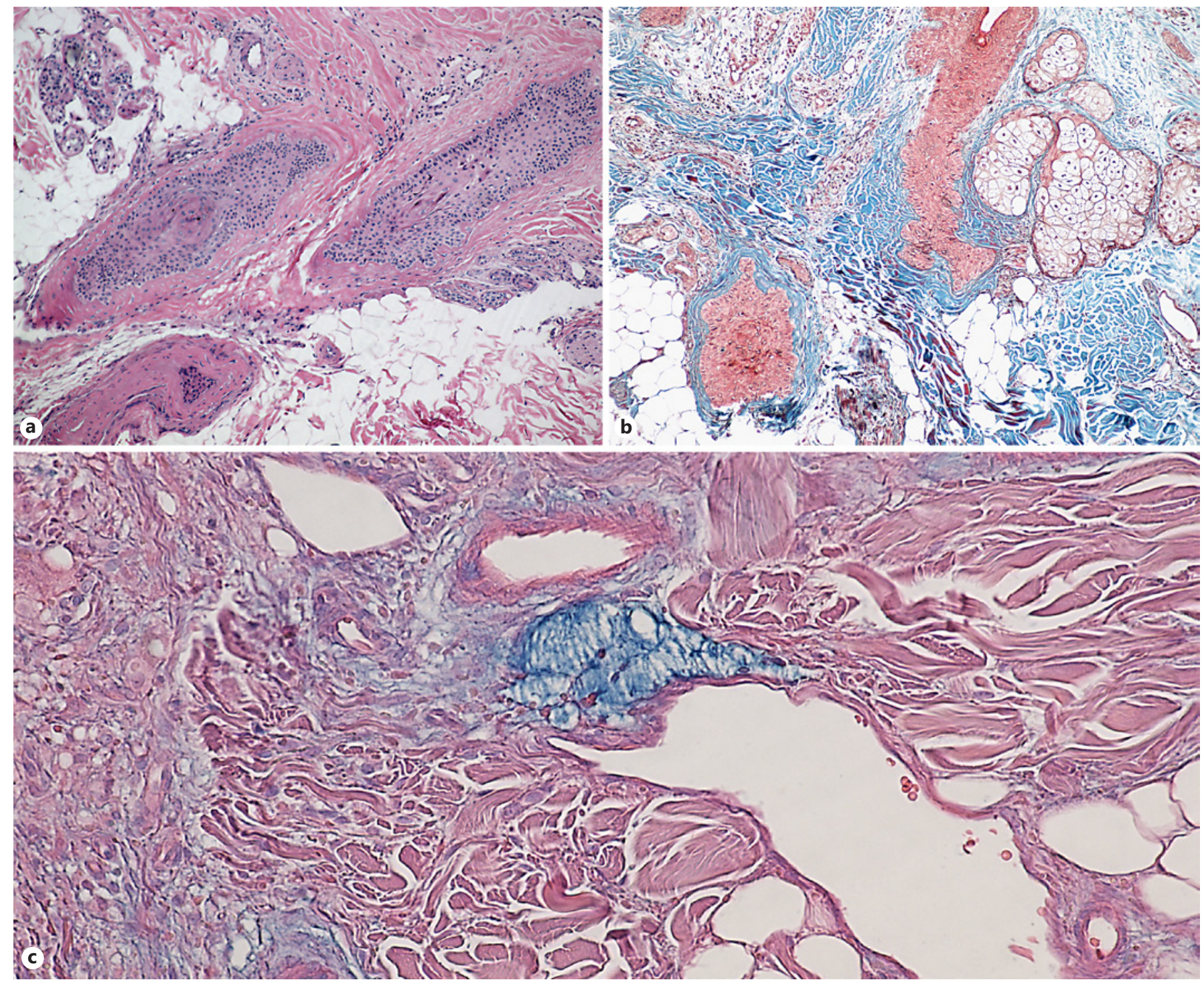

Fig. 3. a Anagen shift with concentric thickening of the perifollicular collagen fibers. $H \& E . \times 10$. b Concentric thickening of perifollicular collagen (Masson's trichrome stain $\times 10$ ). c Intravascular and intradermal HA deposits (Alcian blue stain $\times 40)$.

findings, a vascular occlusion of the supratrochlear artery secondary to HA was suspected. A punch biopsy was performed, and 50 units of hyaluronidase were administered throughout the patch, followed by $100 \mathrm{mg}$ of acetylsalicylic acid PO daily. The patient denied any visual alterations.

The scalp biopsy revealed a decrease in the density of anagenphase hair follicles, dilated capillaries, and a moderate perifollicular inflammatory cell infiltrate composed of lymphocytes and histiocytes. Concentric thickening of the perifollicular collagen fibers was identified (Fig. 3a, b). A basophilic amorphous material within the vessels' lumen and in the dermis causing a granulomatous reaction was observed. This material was positive for alcian blue and colloidal iron stains, indicating that the substance was compatible with HA (Fig. 3c).
One week after the hyaluronidase injection, the alopecic patch stabilized and stopped expanding; swelling, erythema, and pain also decreased. Minoxidil 2\% twice per day was initiated. Upon follow-up 2 months later, hair regrowth was noted, and the erythema, swelling, and trichoscopic findings disappeared (Fig. 4).

\section{Discussion}

Alopecia secondary to HA injection is an underreported complication with only a couple of cases described so far $[3,4]$. It is thought to occur secondary to vascular com- 

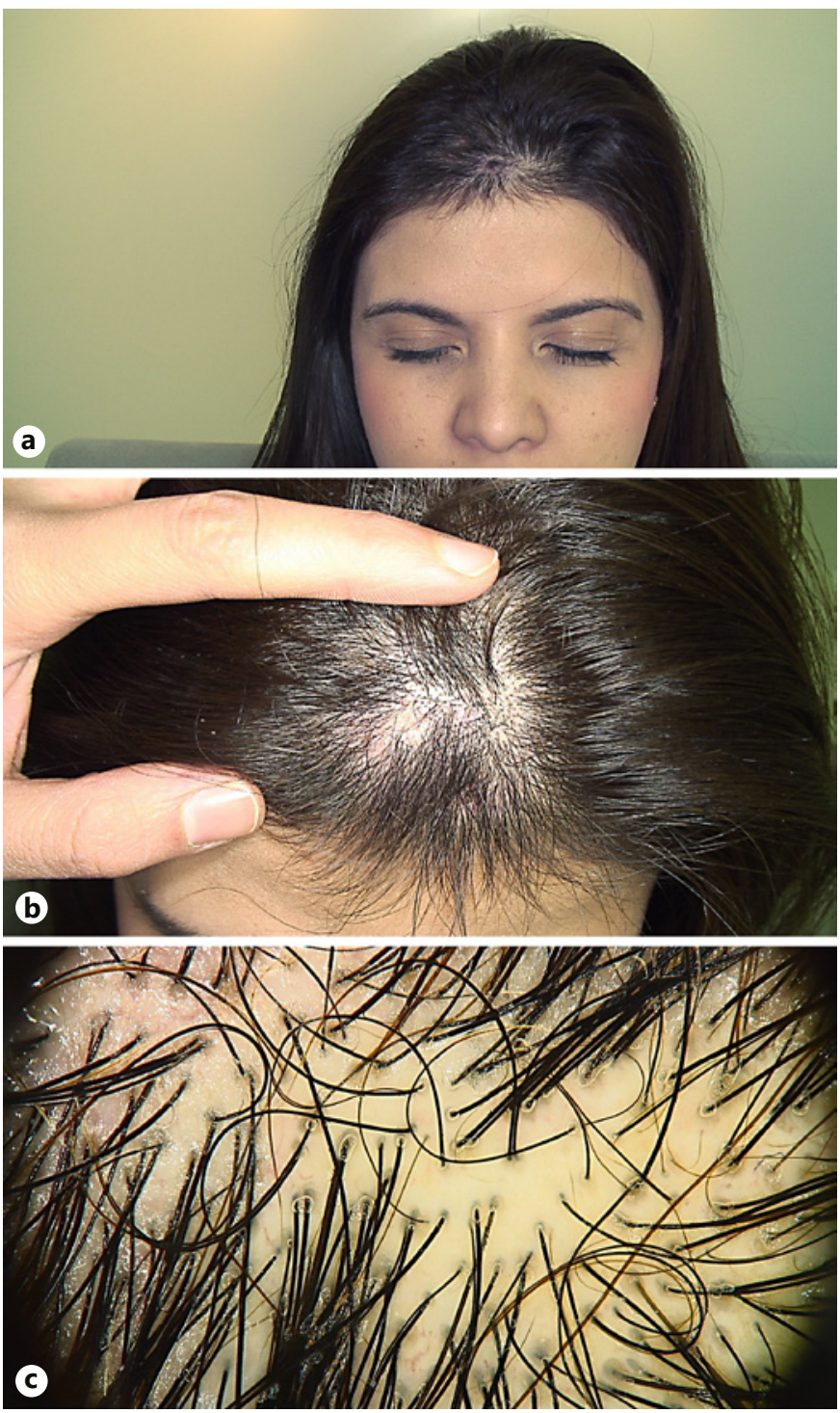

Fig. 4. Follow-up 2 months later showing hair regrowth (a, b) and normalization of the trichoscopic findings (c).

promise, either due to excessive filler volume resulting in vascular tamponade or by embolization of HA leading to intravascular occlusion; both resulting in tissue hypoxia [3]. There are numerous reports of vascular occlusion caused by other types of fillers (calcium hydroxyapatite, poly-L-lactic acid) [5, 6] but no cases of induced alopecia.

Gan et al. [3] and Yang et al. [4] reported two cases of clinical and histopathological findings similar to the one reported in this paper. These authors described nonscarring alopecia resulting from a spontaneous shift from the anagen phase to the telogen phase. This shift was considered to be a consequence of ischemia induced by vascular occlusion due to HA deposits. This sudden disruption of the anagen phase of the hair cycle secondary to hypoperfusion has also been described in pressure-induced alopecia $[7,8]$. The concentric thickening of perifollicular collagen seen in the present case had not been reported before, and it is suggested to be a consequence of the sudden anagen disruption.

Trichoscopic findings in this case can be confused with alopecia areata, anagen effluvium, and noncicatricial pressure-induced alopecia: hairs shed and break (black dots and broken hairs), leaving empty follicles filled with sebum and keratin (yellow dots). Papaiordanou et al. [8] described that trichoscopic features of pressure-induced alopecia are similar to those of alopecia areata. In both entities black dots, broken, and dystrophic hairs can be observed [9]. However, the prominent vascular component seen on trichoscopy of alopecia secondary to HA is a crucial diagnostic clue suggesting a vascular occlusion etiology, as it may correspond to the dilated ectatic vessels founded on histopathology.

Hair regrowth seems to be dependent on whether the ischemia is mild or severe and how promptly the ischemia is reversed [4]. Several treatment options for vascular reperfusion have been described, including hyaluronidase injection, intralesional triamcinolone, nitroglycerin patch, aspirin, and hyperbaric oxygen [3].

Prompt injection of hyaluronidase is the most effective treatment for vascular compromise secondary to HA filler application. However, the optimal time frame for its application to prevent cicatricial alopecia is unknown. In this case, the benefits of hyaluronidase injection were observed even 1 week after the ischemic symptoms. Yang et al. [4] also observed a positive clinical response 9 days later.

\section{Conclusion}

The present report is the first case describing the trichoscopic findings in HA-induced alopecia. This is a type of nonscarring alopecia probably caused by vascular occlusion and secondary disruption of the anagen phase due to tissue hypoxia. The trichoscopic findings include yellow dots, black dots, broken hairs, and ectatic vessels. The prominent vascular component is a trichoscopic clue suggestive of a vascular occlusion etiology.

\section{Statement of Ethics}

This article complies with the guidelines for human studies, and the patient gave her informed consent to publish her case, including publication of images. 


\section{Disclosure Statement}

The authors have no conflicts of interest to declare.

\section{Author Contributions}

Daniel Asz-Sigall: attending physician, analysis, and interpretation of trichoscopic findings, revision of the manuscript, and final approval of the version to be published.
Karla Iñigo-Gomez: writer of the manuscript and collection of data.

María Fernanda Ortega-Springall: writer of the manuscript and collection of data.

María Elisa Vega-Memije: dermatopathologist responsible for the interpretation of the biopsy specimen and histological images, and revision of manuscript.

Roberto Arenas: revision of intellectual content and final approval of the version to be published.

Antonella Tosti: Interpretation of trichoscopic findings, revision of intellectual content, and final approval of the version to be published.

\section{References}

1 Sun ZS, Zhu GZ, Wang HB, Xu X, Cai B, Zeng $\mathrm{L}$, et al. Clinical outcomes of impending nasal skin necrosis related to nose and nasolabial fold augmentation with hyaluronic acid fillers. Plast Reconstr Surg. 2015 Oct;136(4): 434e-41e.

2 Fagien S. Commentary on a Rethink on Hyaluronidase Injection, Intra-arterial Injection and Blindness. Dermatol Surg. 2016 Apr; 42(4):549-52.

3 Gan SD, Itkin A, Wolpowitz D. Hyaluronic acid-induced alopecia: a novel complication. Dermatol Surg. 2013 Nov;39(11):1724-5.
4 Yang Q, Qiu L, Yi C, Xue P, Yu Z, Ma X, et al. Reversible Alopecia with Localized Scalp Necrosis After Accidental Embolization of the Parietal Artery with Hyaluronic Acid. Aesthetic Plast Surg. 2017 Jun;41(3):695-9.

5 Chou CC, Chen HH, Tsai YY, Li YL, Lin HJ. Choroid vascular occlusion and ischemic optic neuropathy after facial calcium hydroxyapatite injection- a case report. BMC Surg. 2015 Mar;15(1):21.

6 Roberts SA, Arthurs BP. Severe visual loss and orbital infarction following periorbital aesthetic poly-(L)-lactic acid (PLLA) injection. Ophthal Plast Reconstr Surg. 2012 May-Jun; 28(3):e68-70.
7 Hanly AJ, Jorda M, Badiavas E, Valencia I, Elgart GW. Postoperative pressure-induced alopecia: report of a case and discussion of the role of apoptosis in non-scarring alopecia. J Cutan Pathol. 1999 Aug;26(7):357-61.

8 Papaiordanou F, da Silveira BR, Piñeiro-Maceira J, Pirmez R. Trichoscopy of noncicatricial pressure-induced alopecia resembling alopecia areata. Int J Trichology. 2016 AprJun;8(2):89-90.

9 Freites-Martinez A, Shapiro J, Goldfarb S, Nangia J, Jimenez JJ, Paus R, et al. Hair disorders in patients with cancer. J Am Acad Dermatol. 2019 May;80(5):1179-1196. 